\title{
COMMENTARY
}

\section{Video laryngoscopy improves intubation success and reduces esophageal intubations compared with direct laryngoscopy in the medical intensive care unit}

Thomas C Mort ${ }^{1,2}$

See related research by Mosier et al., http://ccforum.com/content/17/5/R237

\begin{abstract}
Urgent and emergent airway management outside the operating room is fraught with complications due to the nature of its acuity, single or multiple system dysfunction or failure, and physiological disturbances. These provide a challenge to the airway team and place the patient at grave risk for potentially lifethreatening airway and hemodynamics-related consequences. Conventional laryngoscopy is rapidly being challenged by video-camera-assisted laryngoscopes that, in many cases, offer improved visualization of the airway. Successful intubation remains a lofty but attainable goal for airway specialists as well as the novice intubator. Yet to assume that airway management difficulties can be erased by incorporating a new device is optimistic but naive. In regard to patient safety, the device is just one piece of the airway puzzle.
\end{abstract}

In the previous issue of Critical Care, Mosier and colleagues [1] provide additional support to the growing body of evidence that using video laryngoscopy (VL) improves the first-pass and overall success rate of urgent/ emergent tracheal intubation as well as lowering the incidence of esophageal intubation when compared with conventional Macintosh (curved blade) direct laryngoscopy (DL). They, like others, have found that videoassisted visualization affords a higher percentage of patients with a Cormack-Lehane I/II view and assists the

Correspondence: thomas.mort@hhchealth.org

'Surgical Intensive Care Unit, Hartford Hospital, 80 Seymour Street, Hartford, CT 06102, USA

${ }^{2}$ University of Connecticut School of Medicine, 263 Farmington Avenue, Farmington, CT 06030, USA operator in achieving a higher percentage of glottis opening visualized, thus leading to an improved 'ultimate' success rate when compared with DL [2-4]. Their detailed analysis of a complicated clinical situation acutely ill, critical care patients who required urgent tracheal intubation in a medical intensive care unit setting by providers with a variable levels of experience and skill - is an area uncommonly reported in the literature but often represents the care provided when the anesthesia team is not the primary operator [5-7]. I applaud the consistent imperative toward the improvement of patient safety by this group; each intubation was conducted under attending intensivist supervision [8].

The shortcomings and limitations of DL, our 'gold standard, in the ultra-high risk critically ill population who require urgent/emergent tracheal intubation are, again, on display. The congruent 'line of sight' and the path for tube insertion limit DL visualization and thus its success. VL typically provides an equal or improved view when compared with DL. However, laryngeal exposure is regarded as the simple portion of the VL process. Intubation, even in skilled hands, may be more challenging or impossible, as success relies more heavily on the operator's skill and the patient's airway characteristics than the device itself $[3,5,9,10]$. Understanding that C-Mac (Karl Storz, Tuttlingen, Germany) and GlideScope (Verathon Inc., Bothell, WA, USA) technology possess divergent mechanical and optical complexities based on differing blade design should be highlighted here $[9,11]$. Combining them under a single VL category, as was done in this study, ignores these significant differences. Therefore, VL deployment must be accompanied by diligent and continuous training, especially in light of the rotating house staff. Simply placing VL equipment 
in-service without proper education is a disservice to both the patient and the operator-trainee [12,13]. Proper pre-use instruction, hands-on demonstration, and simulation lab practice should be encouraged.

First-attempt success is a worthy focus of research and a reasonable outcome measurement frequently used in comparative studies of different intubation devices. This is based on the understanding that complications are tempered when fewer intubation attempts are required [13]. Interestingly, this study measured 'ultimate' intubation success with the initial device regardless of the number of attempts ( 3 or more) required by the supervised trainee. Current airway management philosophy advocates a limit on laryngoscopic attempts and favors the rapid enlistment of an alternative management method in the hopes of limiting repetitive intubation attempts, thus reducing adverse airway and hemodynamic consequences [14]. The authors did not report what alternative rescue methods were either made available or used to account for those not ultimately successful. I would opine that, in a general sense and especially in a teaching environment, 'ultimate' success with a single device should be discouraged if it requires continued attempts. More appropriately, early use of an alternative rescue device - that is, laryngeal mask airway, bougie, fiber optic bronchoscopy or VL $\leftrightarrow$ DL - is warranted [12-14]. The less experienced trainee should be taught that early deployment of a rescue device is 'smart' as focusing one's efforts on 'ultimate success with a single device' may turn out to be 'insane'.

Mosier's group raises an important question of whether VL technology can narrow the gap of success between experienced operators and novice trainees. I believe these authors and others are unraveling the evidence that this, indeed, is the case. VL is a significant leap forward toward improving patient safety, yet VL is only one piece of the airway puzzle and we must not become mesmerized by or solely reliant on its past successes. In the post-honeymoon' period after VL deployment, users undergo 'drift' away from adherence to airway fundamentals as their confidence mounts with their VL experience. An experienced airway manager realizes it is far easier to avoid trouble than to try to get out of it. In many cases, VL may compensate for sloppiness and 'drift' from airway fundamentals, but one should choose being 'good' over being 'lucky'. Employing management fundamentals such as proper positioning, that is, ramping for the obese, judicious administration of sedative-analgesics versus the rapid sequence approach, and maintaining spontaneous ventilation when appropriate and an awake technique when indicated for the ultra-high risk patient, is imperative [14]. Airway education should emphasize how to recognize difficult airway characteristics, should teach primary and rescue strategies, and should endorse synchronized and effective written and verbal communication between care providers and should recommend that the most experienced practitioners supervise the intervention, if not perform it, when appropriate. Patient characteristics coupled with operator experience, judgment, and skill lead to or away from intubation success.

As challenging as emergency airway management can be, we must do our best to teach those who will one day be responsible for it and equip them well. VL technology is a welcomed adjunct to the airway puzzle, yet continued vigilance toward its implementation into one's practice is required to maximize its usefulness regardless of the level of the operator's experience. Paraphrasing Einstein, knowledge is power and knowing the how and how not, the dos and don'ts, and when and when not to incorporate $\mathrm{VL}$ is just half the battle.

\section{Abbreviations}

DL: Direct laryngoscopy; VL: Video laryngoscopy.

\section{Competing interests}

The author declares that he has no competing interests.

Published: 03 Dec 2013

\section{References}

1. Mosier JM, Whitmore SP, Bloom JW, Snyder LS, Graham LA, Carr GE, Sakle $J C$ : Video laryngoscopy improves intubation success and reduces esophageal intubations compared to direct laryngoscopy in the medical intensive care unit. Crit Care 2013, 17:R237.

2. Kory P, Guevarra K, Mathew JP, Hegde A, Mayo PH: The impact of video laryngoscopy use during urgent endotracheal intubation in the critically ill. Anesth Analg 2013, 117:144-149.

3. Griesdale DE, Liu D, McKinney J, Choi PT: Glidescope ${ }^{\circledast}$ video-laryngoscopy versus direct laryngoscopy for endotracheal intubation: a systematic review and meta-analysis. Can J Anaesth 2012, 59:41-52.

4. Noppens RR, Geimer S, Eisel N, David M, Piepho T: Endotracheal intubation using the C-MAC(R) video laryngoscope or the Macintosh laryngoscope: a prospective, comparative study in the ICU. Crit Care 2012, 16:R103.

5. Martin LD, Mhyre JM, Shanks AM, Tremper KK: 3,423 emergency tracheal intubations at a university hospital: airway outcomes and complications. Anesthesiology 2011, 114:42-47.

6. Sakles JC, Mosier JM, Chiu S, Keim SM: Tracheal intubation in the emergency department: a comparison of GlideScope((R)) video laryngoscopy to direct laryngoscopy in 822 intubations. J Emerg Med 2012, 42:400-405.

7. Simpson GD, Ross MJ, McKeown DW, Ray DC: Tracheal intubation in the critically ill: a multi-centre national study of practice and complications. Br J Anaesth 2012, 108:792-799.

8. Schmidt UH, Kumwilaisak K, Bittner E, George E, Hess D: Effects of supervision by attending anesthesiologists on complications of emergency tracheal intubation. Anesthesiology 2008, 109:973-977.

9. Behringer EC, Cooper RM, Luney S, Osborn IP: The comparative study of video laryngoscopes to the Macintosh laryngoscope: defining proficiency is critical. Eur J Anaesthesiol 2012, 29:158-159.

10. Cook T, Behringer EC, Benger J: Airway management outside the operating room: hazardous and incompletely studied. Curr Opin Anaesthesiol 2012, 25:461-469.

11. Levitan RM, Heitz JW, Sweeney M, Cooper RM: The complexities of tracheal intubation with direct laryngoscopy and alternative intubation devices. Ann Emerg Med 2011, 57:240-247.

12. Levitan RM: Video laryngoscopy, regardless of blade shape, still requires a backup plan. Ann Emerg Med 2013, 61:421-422.

13. Mort TC: Emergency tracheal intubation: complications associated with repeated laryngoscopic attempts. Anesth Analg 2004, 99:607-613. 
14. Apfelbaum JL, Hagberg CA, Caplan RA, Blitt CD, Connis RT, Nickinovich DG, Hagberg CA, Caplan RA, Benumof JL, Berry FA, Blitt CD, Bode RH, Cheney FW, Connis RT, Guidry OF, Nickinovich DG, Ovassapian A, American Society of Anesthesiologists Task Force on Management of the Difficult Airway: Practice Guidelines for management of the difficult airway: an update report by the ASA. Anesthesiology 2013, 118:251-270.

\section{$10.1186 / \mathrm{cc} 13136$}

Cite this article as: Mort: Video laryngoscopy improves intubation success and reduces esophageal intubations compared with direct laryngoscopy in the medical intensive care unit. Critical Care 2013, 17:1019 